have such restricted beginnings has vital implications. It means that once these original outbreak areas are known they can be kept under proper observation, and any tendency towards the formation of incipient swarms can be dealt with before such swarms can spread and cause widespread devastation. It cannot be too strongly emphasized that Dr. Uvarov himself is the guiding agent in all this international locust policy, as it was his original phase theory that provided the scientific basis for the new outlook on the problem as a whole.

\section{STUMP PRODUCTION IN MADRAS TEAK NURSERIES}

W HAT has come to be known as stump planting, that is, the use of root and shoot cuttings, has been long known in India, some of the earliest experiments made in this direction having been successfully attempted with the sissu (Dalburgia Sissoo) in the irrigated plantations in the Punjab. In Indian Forest Records, 4, No. 5, 1941 (Manager, Government of India Press, New Delhi, 1942), A. L. Griffith says that the planting of stumps in Madras displaced direct dibbling of seed and transplanting, as had been done up to that time. In the early days of stump planting in the presidency a number of experimental investigations were undertaken and continued over some years. Mr. Griffiths summarizes the results in the following tentative conclusions for work in a general West coasts type of climate :

(a) The best density of sowing to use is that of $1 / 8 \mathrm{lb}$. to $1 / 5 \mathrm{lb}$. of teak seed per square foot of nursery bed, and to do no pricking out of seedlings at all. This is most economical in quantity of seed and results in a high proportion of the stumps produced being of the best size for planting at 1 year old (that is, $0.4-0.8 \mathrm{in}$. diameter). (b) Thus, one standard $40 \mathrm{ft} . \times 4 \mathrm{ft}$. nursery bed sown evenly with $20-30 \mathrm{lb}$. of seed on the average produces enough well-shaped stumps of usable size to plant 1 acre of plantation at a $6 \mathrm{ft}$. $\times 6 \mathrm{ft}$. espacement and also leaves a reserve for casualty replacement. (c) Poor seed years have to be guarded against, and at the end of the first year either the undersized plants can be left in the bed and these together with germination from seed still dormant will produce a full bed of goodsized plants at the end of the second year, or the undersized plants can be pulled out with the bigger ones, stumped, and put back into a reformed nursery bed. These plants at the end of the second year will give a high proportion of well-shaped, good-sized stumps which are in no way inferior to fresh, goodsized one year old stumps. (d) Although teak stumps store well, and travel well, a large central permanent nursery for the production of stumps for an extensive area does not appear advisable, as in a few years stump production falls off seriously in most localitios as a result of continued cropping, and this falling off was not prevented by manuring with artificial manure, or by fallowing, or by growing a green manure crop of sunn hemp in alternate years. In addition, pest attack by cockchafer and white ants may be serious in a permanent nursery and occurred in two out of the five years of experiment.

The conclusion arrived at between the permanent and the temporary or flying nursery is most important and of high interest,

\section{ANALYSIS OF ELECTRICAL CONTACT EFFECTS}

TN a paper entitled "Contact Non-Linearity, with reference to the Metal Rectifier and the Carborundum Ceramic Non-Linear Resistor" (J. Inst. Elec. Eng., 89, Pt. 1, No. 23; November 1942), A. Fairweather gives an account of an experimental analysis designed to correlate and formulate the behaviour of various structures possessing contact non-linearity. New data are presented concerning representative contact combinations such as various carborundum combinations, the carborundum ceramic non-linear resistor, the cuprous oxide and selenium rectifiers, and the diamond. Measurements have been made of the variation of the characteristic para. meters, resistance and capacitance, with each of the four significant variables-current, mechanical pressure, thickness of semi-conductor and temperature. The ranges of current and voltage far exceed those which have been employed hitherto. Examination of the results obtained leads to various conclusions regarding the mechanism of contact non-linearity, but not, however, in conflict with the familiar 'barrier layer' hypothesis, which is fully supported by the work described.

It would seem that three clearly defined regions of operation hold for conduction in both directions through a contact possessing contact non-linearity. For 'small' voltages and for 'large' voltages conduction is ohmic: for 'medium' voltages, which are largely those of interest, conduction is non-linear, and obeys a simple empirical equation of the form $V=K i^{n}$, where $n$ depends mainly on the materials, and $K$ on the dimensions, of the contact combination. This type of law might hold for all contact combinations, and its importance is such as to warrant the development of a theory of circuits containing a nonlinear resistor having such a characteristic. Since the contact is never likely to be perfect, the current will be concentrated at a number of spots which may be regarded as imperfections in the boundary. 'The 'small' voltage ohmic resistance is thus identified as a constant resistance arising from spreading current paths associated with these spots. The 'large' voltage ohmic resistance is due to the resistance of the bulk electrodes, which becomes all-important when the boundary resistance has become sufficiently small. The 'medium' voltage non-linear resistance results from conduction across the nominally insulating portion of the boundary.

The simple gap picture of the boundary seems to have been discarded prematurely. In the case of the cuprous oxide rectifier, where it is generally supposed that the boundary layer consists of highly insulating, oxygen-free cuprous oxide, such gaps might result from intercrystalline or crystalline cracking at the boundary, arising during cooling from the high temperature of preparation, and this picture is in closer agreement with the results of experiment. The importance of two much neglected aspects of contact non-linearity are stressed: first, the capacitance parameter, as a possible source of information regarding the insulating portions of the boundary layer, and secondly, the necessity for using structures which are previously unstressed mechanically, for detailed study.

Carborundum, euprous oxide, and selenium are all shown to be ohmic conductors, in opposition to the views of some workers. The tests on contacts between 